\title{
Review
}

\section{What People with Parkinson's Disease Want}

\author{
John Andrejack ${ }^{\mathrm{a}, *}$ and Soania Mathur ${ }^{\mathrm{b}}$ \\ ${ }^{a}$ Queens College, Director of Student Advocacy; Parkinson's Foundation, Patient Advocate In Research, \\ Flushing, NY, USA \\ ${ }^{\mathrm{b}}$ Unshakeable MD, Toronto, ON, Canada
}

Accepted 10 June 2020

\begin{abstract}
Parkinson's disease is an incurable, progressive neurodegenerative disease. This condition is complicated by the varying symptoms in individuals who differ in age of onset, symptoms, progression of disease, response to treatment and prognosis. In this paper, we focus on quality of life achieved through a combination of comprehensive health care, continuous support, and self care. Determining what people with Parkinson's disease want is like assembling multiple puzzles simultaneously. While we surmise that patient centered care, support programs, access to comprehensive health care, and relevant symptom control are pieces of this puzzle, more longitudinal studies-which are observational in nature and correlate the impact of symptoms with patients' reported needs—are necessary.
\end{abstract}

Keywords: Patient-centered care, quality of life, self-care, fatigue, sleep wake disorders, movement disorders

\section{INTRODUCTION AND HOPE FOR A CURE}

An estimated 6 million people globally have been diagnosed with Parkinson's disease, a progressive neurodegenerative disease for which there is currently no cure [1]. Complicating this illness further are its diverse clinical manifestations. The Parkinson's community comprises individuals who differ in terms of age of onset, symptoms, progression of disease, response to treatment and prognosis. Because of this heterogeneity and how individual life experiences impact patients, their expectations and specific treatment goals also vary. Regardless, all patients share one hope- a cure. Until that day, in the words of author Joseph Conrad, "The question is not how to get cured, but how to live." It is quality of life that is most important.

\footnotetext{
${ }^{*}$ Correspondence to: John Andrejack, Queens College, Director of Student Advocacy; Parkinson's Foundation, Patient Advocate In Research, 65-30 Kissena Boulevard, Flushing, NY 11218, USA. Tel.: +1 718997 4486; E-mail: John.Andrejack@qc.cuny.edu.
}

\section{QUALITY OF LIFE}

Quality of life (QoL) may be defined as the perceived quality of a person's daily life, including the physical, emotional and social aspects of their experience. A chronic illness's effect on a person's quality of life can be multidimensional, encompassing physical and occupational function, psychological state, social interaction and somatic sensation.

In Parkinson's disease, an array of features can impact QoL. Studies have shown that symptoms that affect participation in society, limit mobility and prevent independent performance of daily living activities, have the greatest negative impact [2]. Depression, more than the impairment brought on by motor symptoms, is also detrimental to patients' life experience if not appropriately addressed [3].

To best impact patient care, it is important for clinicians to recognize this type of information. We believe that the factors patients need to achieve good QoL are multifaceted. They include quality health care delivered through a patient-centered approach to address the most bothersome and impactful 


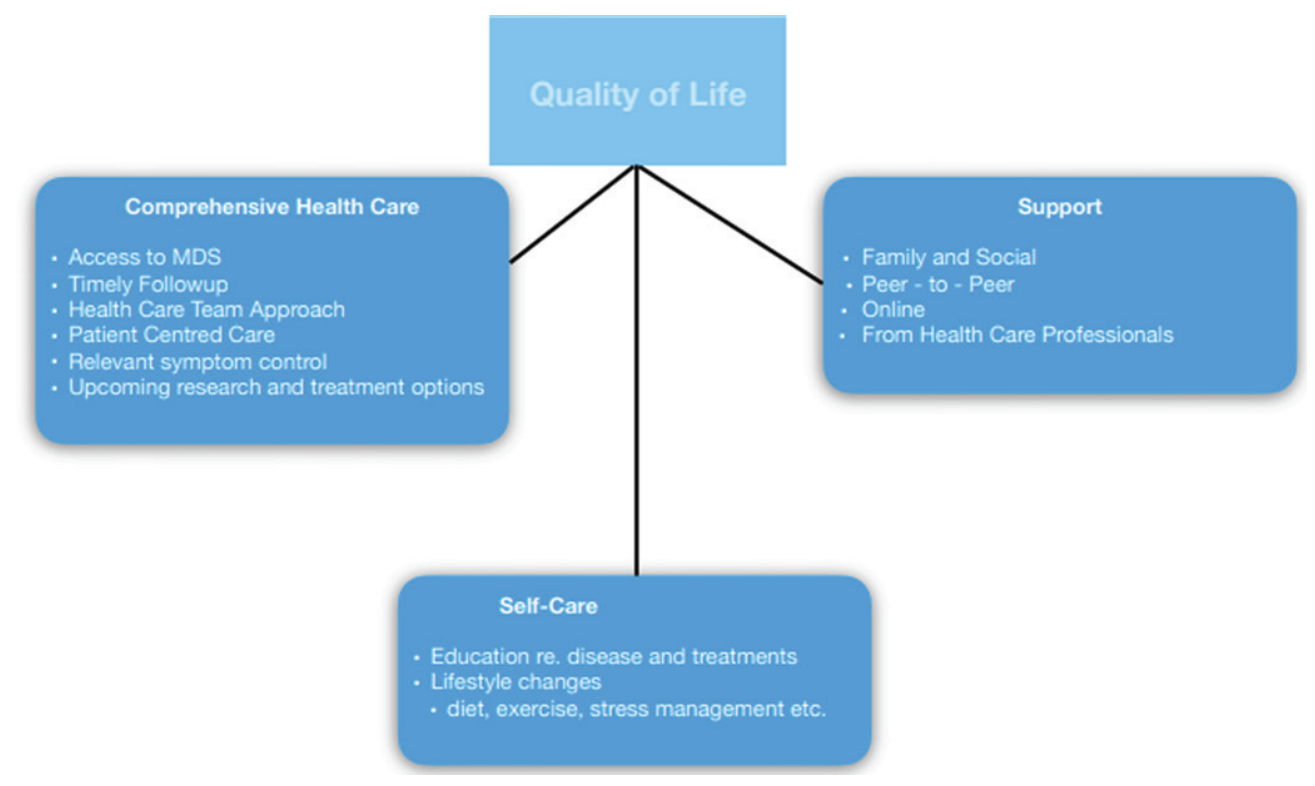

Fig. 1. Factors Contributing To QoL.

symptoms $[2,4,5]$, guidance to establish a comprehensive self-care regimen [6] and significant support from family and community [7] (Fig. 1).

\section{COMPREHENSIVE HEALTH CARE}

What does quality health care mean for patients? People with Parkinson's (PwP) need access to movement disorder specialists, timely follow-up, and patient-centered, multidisciplinary team approaches to their management. A study that explored Parkinson's patient needs from a customer perspective found that the most important requests were: (1) self-management; (2) improved interdisciplinary collaboration of health care professionals; (3) additional time to discuss possible future scenarios; and (4) one health care professional as a single point of contact [8].

The term patient-centered care was introduced to direct the focus from diseases and physicians to patients, their needs and their life experience with their illness $[4,9]$. Physicians seem to view health in terms of bodily functions whereas for patients the definition of health is broader, encompassing mental well-being, spiritual health, quality of life, social participation and daily functioning [10]. In patient-centered care, the person with Parkinson's is the central focus and health care professionals work collaboratively to design a treatment plan for that individual [5]. The degree to which health care decisions are shared between physician and patient as well as the type of decision being made, for example medication treatment versus lifestyle changes, may vary depending on the patient's demographics, education level and stage of disease. But the overall direction must be based on mutual agreement, respect and partnership [11].

Due to the complex variety of motor and non-motor symptoms that characterize Parkinson's disease, this type of care should incorporate different allied health care providers. A multidisciplinary treatment approach has been shown to lead to marked improvement in patient outcomes and better quality of life for patients $[11,12]$.

The health care professional coordinating this multidisciplinary approach will vary depending on location and accessibility [13]. However, the best patient outcomes, especially in terms of managing nonmotor symptoms, off-periods and medication titration, are often achieved with a Movement Disorder Specialist [14]. Yet in early 2014, a survey of Parkinson's disease patients and their families, conducted by The Michael J. Fox Foundation and Abbvie, showed that only $48 \%$ of patients could identify the role of a Movement Disorder Specialist and that only 28\% were being seen by this type of physician [15].

A complex number of features can contribute to reduced QoL in PwP. An imbalance often exists between features that are most closely associated with poor QoL, and those that have been most extensively 
investigated. Poor QoL is most strongly associated with reduced participation in societal roles, followed by the inability to adequately perform daily living activities [16].

Non-motor symptoms are often the first signs of Parkinson's disease and are present in almost all patients, leading to considerable distress and correlating with a negative QoL [17]. However, unlike the disease's four most measured symptoms- tremor, bradykinesia, postural instability and rigidity [18, 19], non-motor symptoms in Parkinson's disease are not easily quantified, diagnosed or treated. In two recent review articles, almost 50\% of respondents reported that non-motor symptoms presented the greatest challenge $[19,20]$ and had the most impact on QoL [19]. While tremors, postural instability and gait issues are often reported by patients, fatigue or lack of energy and pain are also often in the top five most reported motor and non-motor symptoms [19, 21].

Fatigue, sleep disorders and pain are three commonly reported non-motor symptoms that can impact QoL for PwP. Fatigue is one of the most reported nonmotor symptoms [19] and, in some studies, ranks in the top three most reported symptoms overall [17, 19]. A meta-analysis of 44 studies showed that fatigue was prevalent in almost $50 \%$ of Parkinson's disease patients [21]. Fatigue has indeed proven to be bothersome [22] and negatively impact patients' daily lives [20], as well as correlate with a negative QoL [19]. Sleep disorders, which include daytime sleepiness, REM sleep disorders and insomnia, are another category of commonly reported non-motor symptoms that are bothersome and negatively impact patients' daily lives [17, 23]. Pain can be another of the most bothersome symptoms [6] and some patients report it as the symptom that most negatively impacts QoL [21]. Treatment of fatigue, sleep disorders and pain is clearly important to PwP.

Other non-motor symptoms that are impactful for patients are neuropsychiatric (such as depression, anxiety, confusion, hallucinations and cognitive impairment) and gastrointestinal problems. Both categories correlate with a negative QoL [19, 21]. Gastrointestinal symptoms are present in up to $66 \%$ of patients and neuropsychiatric issues can occur in up to $30-50 \%$ of those with Parkinson's disease [17, $19,21]$.

Despite the fact that NMS greatly impact QoL for patients, evidence-based treatments for many of those symptoms is lacking. There are far more advances in treatments for motor symptoms or motor complications of dopaminergic therapy [24]. Successful treatment of NMS entails more large-scale, randomized trials of drugs targeting these issues [23].

Progress is being made in the development of treatments to slow, stop or reverse Parkinson's disease. Several pharmaceutical companies have trials for alpha synuclein vaccination programs and research is being conducted in treatments, which will allow neurons to function despite the presence of pathological alpha synuclein [25]. Until there is a cure, however, PwP will remain concerned about quality of life and symptom management. While treatment of tremors, postural instability and gait issues is important to PwP, it is often the non-motor symptoms that negatively impact QoL. Unfortunately, treatment of non-motor symptoms can be challenging. Health care professionals must first determine if the symptom is iatrogenic, caused by motor symptoms, or a comorbid condition [23]. Even when the cause can be determined, the doctor must check for drug interaction and/or antagonism.

\section{SUPPORT}

Parkinson's disease is a highly complex disease that can be very isolating. Social relationships, with family, extended relatives, friends or neighbors contribute significantly to QoL in PwP [26]. Central to these relationships and good QoL is the ability to communicate without deficit and feel accepted [7]. However, the motor symptoms that patients experience can be bothersome and embarrassing. Fears of developing "off periods" and freezing in public, or of falling, are very real. As the disease progresses, facial, vocal and body muscles become affected, leading to mask-like faces and impaired nonverbal expression that make communication more difficult. The relatively common non-motor neuropsychiatric features of anxiety and depression can also lead to social isolation.

Connecting with others who share similar life experiences can be very therapeutic. Peer support in chronic disease management can be obtained through informal interaction within a community of individuals with the same diagnosis or through a more formal connection with a mentor who may have received training through an advocacy or support organization. Both forms of support are based on experiential knowledge; such relationships can serve as valuable resources for patients. The sense of connection, reduced feelings of isolation, sharing of experiences in living with chronic disease and the exchange of 
coping strategies are among the positive outcomes of peer relationships [27]. Although these relationships may develop through in person support groups, the internet has made it easier to find those who share a similar diagnosis and life experience, regardless of geographic location. Online support communities offer the sharing of experiences, opportunity to learn from the knowledge of a diverse group of individuals, the sense of not being alone, and empathy and understanding [28].

\section{SELF CARE}

PwP want education for themselves, their families and health care professionals on the disease, the symptoms, how to recognize them, new treatment options and coping strategies and available lifestyle changes [6]. In one survey of over 700 Parkinson's disease patients and care partners, only $48 \%$ indicated that they had received education on non-motor symptoms [20]. In this same study, 50\% of the patients and care partners reported that their family and friends were unaware of the impact of the disease on their daily lives [20]. There is some evidence that peer-led psycho-educational programs improved exercise behavior and coping mechanisms in PwP [29]. Patients and caregivers also reported the need for education for their health care professionals who were not Parkinson's experts [6]. Patients and caregivers have clearly indicated a desire for more educational programs, which supports the need for additional studies with larger samples to determine what, if any, impact these programs have on QoL.

While food epidemiology and nutrition studies have biases; including, but not limited to issues with comparison between cohorts, selection and volunteer biases and retention problems, initial findings may still be helpful in creating nutritional plans for individual patients [30]. Research indicates that fresh vegetables, fruit, seeds and nuts, non-fried fish, olive and coconut oil, as well as some herbs and spices are associated with reduced rates of disease progression [31]. Conversely, canned fruit and vegetables, fried food, beef, cheese, ice cream, yogurt and soda have been associated with increased rates of disease progression [31]. In turn, progression of both motor and non-motor symptoms has been shown to impact QoL in PwP [32]. Therefore, we recommend educational programs for PwP should cover healthful eating habits and that healthcare profes- sionals consider using the information from these studies to create individual diet plans tailored for each patient.

There are many physical fitness programs such as dance, boxing, tai chi, qi gong, Pilates, yoga, weight training and indoor cycling designed for PwP. Most exercise programs designed for Parkinson's patients are in the "one-size-fits-all" category. Exercise programs that utilize monitoring watches and/or other wearables would allow health care professionals to remotely monitor and alter the duration and intensity of activity to fit the individual patient [33]. The findings of one study indicate that some aerobic activities may attenuate motor symptoms [34]. It is still uncertain whether exercise slows disease progression, has neuroprotective qualities or just strengthens the immune system, enabling patients to better cope; additional studies with larger sample sizes are necessary [35].

PwP want more information on lifestyle changes [6]. Social support programs and an integrative approach to lifestyle management, including exercise, stress reduction and nutrition, may be clinically significant, but more research is necessary. While there is evidence that these lifestyle changes have general health benefits and may strengthen the immune system, longitudinal studies are needed to identify which lifestyle changes affect particular symptoms [32].

\section{CONCLUSION}

Until there is a cure, PwP want an improved quality of life. This can be achieved through access to comprehensive health care as well as programs designed to provide support and information on self-care. It is clear that the most measured symptoms are not always the most bothersome to patients. Nonetheless, we, too, are making a leap of faith in assuming that symptoms, which have been reported to negatively impact quality of life are those for which PwP would want additional research and treatment options. While some studies [6, 17, 22], have surveyed the needs of PwP and caregivers, additional research is necessary. Longitudinal studies-which are observational in nature and correlate the frequency and impact of the various aspects, symptoms and progression of Parkinson's disease with patients' reported needs-are necessary. We must start asking people with Parkinson's what they want. 


\section{CONFLICT OF INTEREST}

The authors have no conflict of interest to report.

\section{REFERENCES}

[1] Dorsey ER, Bloem BR (2018) The Parkinson pandemic-a call to action. JAMA Neurol 75, 9-10.

[2] Soh SE, McGinley JL, Watts JJ, Iansek R, Murphy AT, Menz HB, Huxham F, Morris ME (2012) Determinants of healthrelated quality of life in people with Parkinson's disease: A path analysis. Qual Life Res 22, 1543-1553.

[3] Uitti RJ (2012) Treatment of Parkinson's disease: Focus on quality of life issues. Parkinsonism Relat Disord 18 (Suppl 1), S34-S46.

[4] Gerteis M, Edgman-Levitan S, Daley J, Delbanco TL (1997) Through the patient's eyes: Understanding and promoting patient-centered care. J Healthcare Quality (JHQ) 19, 43.

[5] Radder DLM, de Vries NM, Riksen NP, Diamond SJ, Gross D, Gold DR, Heesakkers J, Henderson E, Hommel ALAJ, Lennaerts HH, Busch J, Dorsey RE, Andrejack J, Bloem BR (2019) Multidisciplinary care for people with Parkinson's disease: The new kids on the block! Expert Rev Neurother 19, 145-157.

[6] Uebelacker LA, Epstein-Lubow G, Lewis T, Broughton MK, Friedman JH (2014) A survey of Parkinson's disease patients: Most bothersome symptoms and coping preferences. J Parkinsons Dis 4, 717-723.

[7] Takahashi K, Kamide N, Suzuki M, Fukuda M (2016) Quality of life in people with Parkinson's disease: The relevance of social relationships and communication. J Phys Ther Sci 28, 541-546.

[8] Vlaanderen FP, Rompen L, Munneke M, Stoffer M, Bloem BR, Faber MJ (2019) The voice of the Parkinson customer. J Parkinsons Dis 9, 197-201.

[9] Barry MJ, Edgman-Levitan S (2012) Shared decision making-pinnacle of patient-centered care. $N$ Engl J Med 366, 780-781.

[10] Huber M, van Vliet M, Giezenberg M, Winkens B, Heerkens Y, DagneliePC, Knottnerus JA (2016) Towards a "patientcentred" operationalisation of the new dynamic concept of health: A mixed methods study. BMJ Open 6, e010091.

[11] Zizzo N, Bell E, Lafontaine AL, Racine E (2017) Examining chronic care patient preferences for involvement in health-care decision making: The case of Parkinson's disease patients in a patient-centred clinic. Health Expect 20, 655-664.

[12] Bloem BR, Henderson EJ, Dorsey ER, Okun MS, Okubadejo N, Chan P, Andrejack J, Darweesh SKL, Munneke M (2020) Integrated and patient-centred management of Parkinson's disease: A network model for reshaping chronic neurological care. Lancet Neurol, doi: 10.1016/S1474-4422(20)30064-8.

[13] Prizer LP, Browner N (2012) The integrative care of Parkinson's disease: A systematic review. J Parkinsons Dis 2, 79-86.

[14] Cheng EM, Swarztrauber K, Siderowf AD, Eisa MS, Lee M, Vassar S, Jacob E, Vickrey BG (2007) Association of specialist involvement and quality of care for Parkinson's disease. Mov Disord 22, 515-522.

[15] Sawicki K (2015) Survey Reveals Knowledge Gap Among Parkinson's Patients and Caregivers [Internet]. The Michael J. Fox Foundation for Parkinson's
Research | Parkinson's Disease. Available from: https://www.michaeljfox.org/news/survey-reveals-

knowledge-gap-among-parkinsons-patients-and-caregivers

[16] van Uem JM, Marinus J, Canning C, van Lummel R, Dodel R, Liepelt-Scarfone I, Berg D, Morris ME, Maetzler W (2016) Health-Related Quality of Life in patients with Parkinson's disease-A systematic review based on the ICF model. Neurosci Biobehav Rev 61, 26-34.

[17] Breen KC, Drutyte G (2013) Non-motor symptoms of Parkinson's disease: The patient's perspective. J Neural Transm 120, 531-535.

[18] Barone P, Antonini A, Colosimo C, Marconi R, Morgante L, Avarello TP, Bottacchi E, Cannas A, Ceravolo G, Ceravolo R, Cicarelli G, Gaglio RM, Giglia RM, Iemolo F, Manfredi M, Meco G, Nicoletti A, Pederzoli M, Petrone A, Pisani A, Pontieri FE, Quatrale R, Ramat S, Scala R, Volpe G, Zappulla S, Bentivoglio AR, Stocchi F, Trianni G, Dotto PD (2009) The PRIAMO study: A multicenter assessment of nonmotor symptoms and their impact on quality of life in Parkinson's disease. Mov Disord 24, 1641-1649.

[19] Mathur S, Mursaleen L, Stamford J, DeWitte S, Robledo I, Isaacs T (2017) Challenges of improving patientcentred care in Parkinson's disease. J Parkinsons Dis 7, 163-174.

[20] Hermanowicz N, Jones SA, Hauser RA (2019) Impact of non-motor symptoms in Parkinson's disease: A PMDAlliance survey. Neuropsychiatr Dis Treat 15, 2205-2212.

[21] Tarolli CG, Zimmerman GA, Auinger P, McIntosh S, Horowitz RK, Kluger BM, Dorsey ER, Holloway RG (2020) Symptom burden among individuals with Parkinson disease: A national survey. Neurol Clin Pract 10, 65-72.

[22] Siciliano M, Trojano L, Santangelo G, De Micco R, Tedeschi G, Tessitore A (2018) Fatigue in Parkinson's disease: A systematic review and meta-analysis. Mov Disord 33, 1712-1723.

[23] Loddo G, Calandra-Buonaura G, Sambati L, Giannini G, Cecere A, Cortelli P, Provini F (2017) The treatment of sleep disorders in Parkinson's disease: From research to clinical practice. Front Neurol 8, 42.

[24] Titova N, Chaudhuri KR (2017) Nonmotor Parkinson's and future directions. Int Rev Neurobiol 134, 1493-1505.

[25] Foltynie T, Langston JW (2018) Therapies to slow, stop, or reverse Parkinson's disease. J Parkinsons Dis $\mathbf{8}$, S115-S121.

[26] Soundy A, Collett J, Lawrie S, Coe S, Roberts H, Hu M, Bromley S, Harling P, Reed A, Coeberg J, Carroll C, Dawes $\mathrm{H}$ (2019) A qualitative study on the impact of first steps-A peer-led educational intervention for people newly diagnosed with Parkinson's disease. Behav Sci (Basel) 9, 107.

[27] Embuldeniya G, Veinot P, Bell E, Bell M, Nyhof-Young J, Sale JE, Britten N (2013) The experience and impact of chronic disease peer support interventions: A qualitative synthesis. Patient Educ Couns 92, 3-12.

[28] Attard A, Coulson NS (2012) A thematic analysis of patient communication in Parkinson's disease online support group discussion forums. Comput Human Behav 28, 500-506.

[29] Soundy A, Stubbs B, Roskell C (2014) The experience of Parkinson's disease: A systematic review and metaethnography. Scientific WorldJournal 2014, 613592.

[30] Heinzel S, Lerche S, Maetzler W, Berg D (2017) Global, yet incomplete overview of cohort studies in Parkinson's disease. J Parkinsons Dis 7, 423-432.

[31] Mischley LK, Lau RC, Bennett RD (2017) Role of diet and nutritional supplements in Parkinson's disease progression. Oxid Med Cell Longev 2017, 6405278. 
[32] Erro R, Picillo M, Vitale C, Amboni M, Moccia M, Santangelo G, Pellecchia MT, Barone P (2016) The non-motor side of the honeymoon period of Parkinson's disease and its relationship with quality of life: A 4-year longitudinal study. Eur J Neurol 23, 1673-1679.

[33] Dobkin BH (2017) A rehabilitation-internet-of-things in the home to augment motor skills and exercise training. $\mathrm{Neu}$ rorehabil Neural Repair 31, 217-227.
[34] van der Kolk NM, de Vries NM, Kessels RPC, Joosten H, Zwinderman AH, Post B, Bloem BR (2019) Effectiveness of home-based and remotely supervised aerobic exercise in Parkinson's disease: A double-blind, randomised controlled trial. Lancet Neurol 18, 998-1008.

[35] Ellis T, Rochester L (2018) Mobilizing Parkinson's disease: The future of exercise. J Parkinsons Dis 8, S95-S100. 